\title{
Fixed Combination of Travoprost and Timolol Maleate Reduces Intraocular Pressure in Japanese Patients with Primary Open-Angle Glaucoma or Ocular Hypertension: A Prospective Multicenter Open-Label Study
}

Tadashi Nakano $\cdot$ Shiro Mizoue $\cdot$ Nobuo Fuse $\cdot$ Aiko Iwase •

Shun Matsumoto $\cdot$ Keiji Yoshikawa

To view enhanced content go to www.advancesintherapy.com Received: July 23, 2015 / Published online: September 30, 2015

(C) The Author(s) 2015. This article is published with open access at Springerlink.com

\section{ABSTRACT}

Introduction: The efficacy of lowering intraocular pressure (IOP) and safety of switching to travoprost/timolol fixed combination ophthalmic solution $\left(\right.$ Duotrav $^{\circledR}$, Alcon Laboratories, Inc., Fort Worth, TX, USA)

For the I.CHANGE2 Study Group.

IOP: CHecked and Assessed in Normal tension Glaucoma by Exceptional glaucomatologists 2 Study Group (details are included in Electronic Supplementary Material).

Electronic supplementary material The online version of this article (doi:10.1007/s12325-015-0246-9) contains supplementary material, which is available to authorized users.

T. Nakano $(\square)$

Department of Ophthalmology, The Jikei University School of Medicine, Nishi-Shimbashi, Minato-ku,

Tokyo, Japan

e-mail: tnakano@jikei.ac.jp

\section{S. Mizoue}

Department of Ophthalmology, Ehime University Graduate School of Medicine, Shitsukawa, Toon, Ehime, Japan

N. Fuse

Department of Integrative Genomics, Tohoku Medical Megabank Organization, Seiryo-machi, Aoba-ku, Sendai, Miyagi, Japan in patients with primary open-angle glaucoma, normal tension glaucoma or ocular hypertension undergoing prostaglandin analog (PGA) monotherapy was investigated.

Methods: Patients treated with travoprost, latanoprost, tafluprost, or bimatoprost for $\geq 3$ months and requiring additional medication were switched to Duotrav without washout. Baseline IOP was calculated from measurements at two visits during PGA monotherapy. IOP reductions at 4, 8, and 12 weeks after switching to Duotrav and adverse events were assessed.

Results: Of 162 patients enrolled, 157 patients (96.9\%) with $\geq 4$ weeks of follow-up after switching to Duotrav were analyzed. The mean

\author{
A. Iwase \\ Tajimi Iwase Eye Clinic, Honmachi, Tajimi, Gifu, \\ Japan \\ S. Matsumoto \\ Department of Ophthalmology, Tokyo Teishin \\ Hospital, Fujimi, Chiyoda-ku, Tokyo, Japan \\ K. Yoshikawa \\ Yoshikawa Eye Clinic, Nakamachi, Machida, Tokyo, \\ Japan
}


IOP decreased significantly (baseline $=$ $16.3 \pm 3.1 \mathrm{mmHg} ; 4$ weeks $=14.6 \pm 3.1 \mathrm{mmHg}$, 8 weeks $=14.7 \pm 3.3 \mathrm{mmHg}, \quad 12$ weeks $=$ $14.6 \pm 3.2 \mathrm{mmHg}$; all $P<0.0001)$. When study eyes were divided into three groups according to baseline IOP ( $\geq 19 \mathrm{mmHg}$ : 33 eyes, $21.0 \%$; $\geq 15$ to $<19$ mmHg: 78 eyes, 49.7\%; <15 mmHg: 46 eyes, 29.3\%), all groups showed significant IOP reductions $\quad(P=0.0324 \sim P<0.0001) \quad$ after switching to Duotrav. Twenty-seven of 166 patients (16.3\%) in the safety analysis experienced adverse events and 26/166 patients (15.7\%) experienced adverse events, for which a relationship to Duotrav could not be ruled out. Adverse events in five patients led to treatment discontinuation (eye pruritus; eye irritation; increased blood pressure and rash; increased blurred vision; deepening of the eyelid sulcus and blepharoptosis). Twelve weeks after treatment switching, eyelash changes, blepharal pigmentation and deepening of the eyelid sulcus occurred in 42 (26.8\%), 29 (18.5\%), and 13 (8.3\%) cases, respectively, among 157 patients with follow-up. There was no significant worsening from baseline for superficial punctate keratopathy (SPK) or conjunctival hyperemia after switching (SPK score: baseline $=0.58 \pm 1.31 ; 12$ weeks $=$ $0.92 \pm 1.76, P=0.1819$; conjunctival hyperemia score: $\quad$ baseline $=0.41 \pm 0.64 ; \quad 12$ weeks $=$ $0.49 \pm 0.63, P=0.3774)$.

Conclusion: Our findings confirm that switching to Duotrav $^{\circledR}$ in PGA monotherapy patients shows IOP-lowering effect with minimal safety concerns.

Funding: Japan Association of Health Service and Alcon Japan. Ltd.

Trial registration: UMIN Clinical Trials Registry identifier, UMIN000007028.

Keywords: Adverse event; Intraocular pressure; Travoprost/timolol fixed combination; Side effect; Switching therapy

\section{INTRODUCTION}

Lowering intraocular pressure (IOP) is the only evidence-based strategy for managing patients with glaucoma and prostaglandin analogs (PGAs) that effectively lower IOP represent the current first-line topical ophthalmic treatment for the disease [1]. However, visual field defects associated with glaucoma can continue to progress even after IOP is lowered to a specific target level [2]. Nearly, $40 \%$ of patients who have experienced a significant IOP reduction with PGA therapy require a further reduction and start to receive an additional topical ophthalmic medication $[3,4]$. Combination regimens using multiple different ophthalmic solutions are less convenient for patients and increase their burden because patients need to take their prescribed ophthalmic solutions several times a day with a wait of at least $5 \mathrm{~min}$ between taking different solutions [5-7]. In contrast, fixed drugs of a PGA and a $\beta$-blocker produce a similar additive IOP-lowering effect without increasing the burden on patients and thus may be advantageous in preventing the risk of becoming non-adherent after the addition of a second drug [8].

Previous studies have reported significant IOP reductions after switching from PGA monotherapy (latanoprost, travoprost, or bimatoprost) to fixed drugs containing the corresponding PGA [9-11]. However, these studies mainly included patients with open-angle glaucoma with a high baseline IOP (>19 $\mathrm{mmHg}$ ) or those with ocular hypertension $(\mathrm{OH})$. No studies have evaluated the efficacy of travoprost/timolol fixed combination (Duotrav ${ }^{\circledR}$, Alcon Laboratories, Inc., Fort Worth, TX, USA) therapy in patients whose 
IOP has already been lowered to $\leq 19 \mathrm{mmHg}$ with PGA monotherapy [10, 12, 13].

Because visual field defects progress steadily even in normal tension glaucoma (NTG) [14], a condition where IOP remains normal, NTG patients are also treated with PGA-based IOP-lowering therapy [15, 16], and if visual field defects continue to progress despite a significant IOP reduction with initial monotherapy, a second drug is added [17]. Thus, these patients are also potential candidates to receive fixed drugs therapy. However, the efficacy of switching to a fixed drug therapy has not been extensively studied in this patient population.

The four PGAs currently available for the treatment of glaucoma are travoprost, latanoprost, tafluprost, and bimatoprost and for each of these, fixed combination ophthalmic solutions are commercially available or have been approved [18]. Among these solutions, Duotrav which contains polyquaternium-1 (Polyquad $^{\circledR}, \quad$ Alcon Laboratories, Inc., Fort Worth, TX, USA) as a preservative is the only one that does not contain benzalkonium chloride (BAK), a preservative that increases the risk of corneal damage [19], when switching from monotherapy.

In the present study, we investigated whether patients with open-angle glaucoma, including NTG, or with $\mathrm{OH}$ who had been treated with one of the four PGAs for at least 3 months and some of whom had an IOP of less than $19 \mathrm{mmHg}$ would experience a further reduction in IOP after switching to Duotrav. Here, we report the efficacy of Duotrav in this patient population as well as the local and systemic safety results, including effects on the ocular surface.

\section{METHODS}

\section{Subjects}

Patients eligible for the study were aged 20 years or older, had been diagnosed with primary open-angle glaucoma (POAG), NTG, or $\mathrm{OH}$, and were judged by their treating doctors to require additional ophthalmic medication due to the progression of visual field defects or uncontrolled IOP despite continued treatment with one of four PGAs for at least 3 months.

Exclusion criteria: (1) a mean deviation (MD) of less than $-20 \mathrm{~dB}$ by visual field testing; (2) a history of hypersensitivity to $\beta$-blockers or a contraindication to $\beta$-blockers (e.g., bronchial asthma and uncontrolled heart failure); (3) the presence of chronic or recurrent uveitis, scleritis or corneal herpes; (4) a history of ocular trauma, intraocular surgery or laser treatment within 3 months prior to the baseline evaluation; (5) any condition that would preclude accurate IOP measurement by applanation tonometry; (6) the use of ophthalmic corticosteroids; (7) serious ocular complications; (8) pregnancy or lactation; and (9) severe dementia. Patients whose participation in the study was deemed inappropriate by their treating doctors were also excluded. Gender and the presence of dry eyes were not considered when enrolling patients.

Prior to any study procedures, the protocol and all aspects of the study were reviewed and approved by the ethics committee of Kitamachi Clinic, Tohoku University Hospital, Juntendo University Urayasu Hospital, the Jikei University School of Medicine, Nakano General Hospital, Fussa Hospital, Fukui-ken Saiseikai Hospital and Minami-Matsuyama Hospital. This study was registered with the University Hospital Medical Information Network (UMIN), number UMIN000007028. 
Among potential subjects who met all of the inclusion and none of the exclusion criteria, only those who signed informed consent forms through the process according to the principles of the Helsinki declaration were enrolled in the study.

\section{Procedures}

This was a prospective multicenter open-label study. Patients requiring a further IOP reduction in the opinion of their treating doctors were switched from PGA monotherapy to travoprost/timolol maleate fixed combination (Duotrav) without having a period of washout from the previous PGA. One drop of Duotrav was instilled into the conjunctival sac of one or both eyes in the morning once daily. Patients underwent protocol-specified examinations before (at baseline) and at 4, 8, and 12 weeks after switching. In each patient, IOP was measured in duplicate by the same examiner throughout the study period using the Goldmann applanation tonometer and the mean of the two values was used for data analysis. IOP measurements during follow-up were performed at the same time of day $( \pm 2 \mathrm{~h})$ as baseline measurements. During each visit, eyes were examined for superficial punctate keratopathy (SPK) and conjunctival hyperemia by slit-lamp microscopy. The degree of SPK was scored on a 5-point scale from 0 to 4 according to the cornea diagram of the National Eye Institute (NEI) classification system [20]. The degree of conjunctival hyperemia was scored on a 4-point scale, where $0=$ no dilated vessels, $1=$ dilation of primarily small vessels, $2=$ dilation of small and large vessels, and $3=$ significant dilation of small and large vessels. These assessments were rated by reference to sample pictures of eyes with SPK and conjunctival hyperemia that were provided beforehand. To collect data on adverse events, doctors asked patients if they had any symptoms and also examined them for any signs or symptoms during each visit. Patients were always asked whether there was irritation, foreign body sensations, dry feeling or itching in the patient's eyes. Examination for eyelash changes, blepharal pigmentation and deepening of the eyelid sulcus was also mandatory. To detect these adverse events, pictures of eyes were taken every visit and changes from baseline were recorded. Examinations were performed according to each center's practice and instruments, and conditions for taking pictures were not standardized. At the start of Duotrav therapy, patients were instructed to remove any solution spilled on the eyelid skin by wiping or washing their face. At the start and end of the study, systolic/diastolic blood pressure and pulse rate were measured.

\section{Statistical Analysis}

Primary endpoint was IOP, IOP reduction and IOP reduction rate from baseline. Secondary endpoint was safety including the score of SPK and conjunctival hyperemia.

Patient data including disease subtype and IOP measurements were collected at each center were sent to and assembled by Tajimi Iwase Eye Clinic in an anonymous form. An ophthalmologist not involved in data collection (M.S.) validated each case. Patients who completed at least 4 weeks of follow-up were included in the analysis.

Baseline IOP was defined as the mean of two measurements obtained at two visits during PGA monotherapy. If both eyes were eligible for the study, the eye with a higher baseline IOP was chose or, if both eyes had an equal baseline 
IOP, the right eye was selected as the study eye. Absolute and percent IOP reductions from baseline were analyzed for study eyes of patients who were followed up for at least 4 weeks after starting Duotrav therapy using repeated-measures analysis of variance and Steel's multiple comparison test. SPK and conjunctival hyperemia scores were analyzed by Steel's test. Missing values after 4 weeks were imputed using last observation carried forward method.

Adverse events for which a relationship to Duotrav could not be ruled out were graded according to the following criteria: mild (the patient recognized the adverse event but could tolerate the symptom), moderate (the patient found the symptom interfered with normal activities and was uncomfortable) and severe (the symptom was physically disabling and prevented the patient from working and normal activities). Eyelash changes, blepharal pigmentation and deepening of the eyelid sulcus were analyzed separately and cumulative incidence rates were calculated. Overall incidence rate of adverse events was also calculated. Changes from baseline in blood pressure, pulse rate were analyzed using the Kruskal-Wallis test. Statistical analysis was performed by QOL RD Co., Ltd., and SOC Co., Ltd., using JMP ${ }^{\circledR} 9.0$ and $\mathrm{SAS}^{\circledR} 9.3$ (SAS Institute Inc., Cary, NC, USA) with a two-sided significance level of $5 \%$.

\section{RESULTS}

The study was conducted from August 2011 to March 2013. Among 166 patients who gave informed consent for the study, 162 patients (97.6\%) were eligible according to the inclusion and exclusion criteria. Five of these 162 patients dropped out within 4 weeks (one developed eye pruritus, two requested treatment discontinuation and two missed visits); the remaining 157 patients (96.9\%) with at least 4 weeks of follow-up were included in the analysis.

The analysis population consisted of 54 males and 103 females with a mean age of $66.6 \pm 11.3$ years (range $39-88$ years). Of these, 60 (38.2\%) had POAG, 78 (49.7\%) had NTG, and 19 (12.1\%) had $\mathrm{OH}$. There were no significant differences in age or sex between the disease subgroups (Table 1). Ten of 157 patients $(6.4 \%)$ discontinued the study before 12 weeks. Reasons for discontinuation included blurred vision (1), increased blood pressure and rash (1), deepening of the eyelid sulcus and blepharoptosis (1), eye irritation (1), insufficient IOP-lowering effect (1), patient's request (4), and loss to follow-up (1).

After the initiation of travoprost/timolol maleate fixed combination (Duotrav) treatment, the mean IOP of eyes included in the analysis significantly decreased from $16.3 \pm$ $3.1 \mathrm{mmHg}$ at baseline to $14.6 \pm 3.1 \mathrm{mmHg}$ at 4 weeks, $14.7 \pm 3.3 \mathrm{mmHg}$ at 8 weeks and $14.6 \pm 3.2 \mathrm{mmHg}$ at 12 weeks $(P<0.0001$ for all time points). The mean percent reduction in IOP from baseline was $10.3 \pm 12.7 \%, 9.4 \pm$ $14.3 \%$, and $10.1 \pm 13.0 \%$ at 4,8 , and 12 weeks, respectively.

Individual baseline IOP values had a wide range from 9.5 to $27.5 \mathrm{mmHg}$ (interquartile range 14.5 to $18.5 \mathrm{mmHg}$ ) and were $\geq 19 \mathrm{mmHg}$ for 33 eyes $(21.0 \%), \geq 15$ to $<19$ $\mathrm{mmHg}$ for 78 eyes (49.7\%), and $<15 \mathrm{mmHg}$ for 46 eyes (29.3\%; Fig. 1 ). We then assessed the treatment effect by baseline IOP. When study eyes were divided into three groups according to baseline IOP $(\geq 19 \mathrm{mmHg}, \geq 15$ to $<19 \mathrm{mmHg}$, or $<15 \mathrm{mmHg}$ ), all groups had significant IOP reductions from baseline at 4 , 
Table 1 Patient characteristics

\begin{tabular}{|c|c|c|c|c|}
\hline Characteristic & POAG & $\mathbf{O H}$ & NTG & Total \\
\hline$n$ & 60 & 19 & 78 & 157 \\
\hline Male, $n(\%)$ & $14(24.6 \%)$ & $4(21.1 \%)$ & $34(43.6 \%)$ & 54 \\
\hline Female, $n(\%)$ & $43(75.4 \%)$ & $15(78.9 \%)$ & $44(56.4 \%)$ & 103 \\
\hline \multirow[t]{2}{*}{ Age, years } & $n=60$ & $n=19$ & $n=78$ & $n=157$ \\
\hline & $67.5 \pm 11.8(39-88)$ & $68.2 \pm 10.8(47-87)$ & $65.5 \pm 11.2(40-88)$ & $66.6 \pm 11.3(39-88)$ \\
\hline \multicolumn{5}{|l|}{ Previous PGA, $n(\%)$} \\
\hline Travoprost & 23 & 8 & 39 & $70(44.6 \%)$ \\
\hline Latanoprost & 15 & 5 & 25 & $45(28.7 \%)$ \\
\hline Tafluprost & 16 & 4 & 9 & $29(18.5 \%)$ \\
\hline Bimatoprost & 6 & 2 & 5 & $13(8.3 \%)$ \\
\hline \multirow{2}{*}{$\begin{array}{l}\text { Baseline IOP, } \\
\text { mmHg }\end{array}$} & $n=60$ & $n=19$ & $n=78$ & $n=157$ \\
\hline & $17.9 \pm 2.7(12.0-27.5)$ & $\begin{array}{l}18.8 \pm 2.9 \\
\quad(13.5-22.5)\end{array}$ & $14.5 \pm 2.2(9.5-19.5)$ & $16.3 \pm 3.1(9.5-27.5)$ \\
\hline \multirow{2}{*}{$\begin{array}{l}\text { Corneal thickness, } \\
\mu \mathrm{m}\end{array}$} & $n=37$ & $n=7$ & $n=65$ & $n=109$ \\
\hline & $\begin{array}{r}522.4 \pm 35.3 \\
(434-629)\end{array}$ & $\begin{array}{r}542.7 \pm 40.0 \\
(486-589)\end{array}$ & $\begin{array}{r}524.7 \pm 33.1 \\
(454-590)\end{array}$ & $\begin{array}{r}525.0 \pm 34.3 \\
(434-629)\end{array}$ \\
\hline \multirow[t]{2}{*}{$\mathrm{MD}, \mathrm{dB}$} & $n=57$ & $n=19$ & $n=73$ & $n=149$ \\
\hline & $\begin{array}{l}-5.51 \pm 4.95 \\
\quad(-19.49-1.09)\end{array}$ & $\begin{array}{l}0.09 \pm 1.58 \\
\quad(-2.92-2.54)\end{array}$ & $\begin{array}{l}-5.52 \pm 4.77 \\
\quad(-16.06-0.72)\end{array}$ & $\begin{array}{l}-4.80 \pm 4.92 \\
\quad(-19.49-2.54)\end{array}$ \\
\hline \multirow[t]{2}{*}{ PSD, dB } & $n=57$ & $n=19$ & $n=73$ & $n=149$ \\
\hline & $\begin{array}{l}6.63 \pm 4.30 \\
\quad(1.15-14.93)\end{array}$ & $\begin{array}{l}2.36 \pm 1.59 \\
\quad(1.15-6.81)\end{array}$ & $\begin{array}{l}7.12 \pm 4.46 \\
\quad(1.25-16.68)\end{array}$ & $\begin{array}{l}6.33 \pm 4.40 \\
\quad(1.15-16.68)\end{array}$ \\
\hline
\end{tabular}

Data are shown as mean \pm standard deviation (range) unless otherwise stated

$O H$ ocular hypertension, $I O P$ intraocular pressure, $M D$ mean deviation, $N T G$ normal tension glaucoma, $P G A$ prostaglandin analog, $P O A G$ primary open-angle glaucoma, $P S D$ pattern standard deviation

8, and 12 weeks after switching to Duotrav (Fig. 2).

In a multiple regression analysis with IOP reduction from baseline at 12 weeks after switching to Duotrav as a dependent variable and demographic and baseline characteristics (type of prior PGA, age, gender, treatment duration, baseline IOP, MD and pattern standard deviation (PSD) values as independent variables, the type of PGA used in prior treatment and baseline IOP had a significant association (Table 2).

There was no significant worsening of SPK total scores or conjunctival hyperemia scores after switching to Duotrav (Table 3). During the study, 26 of 166 patients (15.7\%) experienced 42 adverse events for which a relationship to Duotrav could not be ruled out. Among the 42 adverse events, 38 in 24 patients were mild in severity. The remaining four events in four 


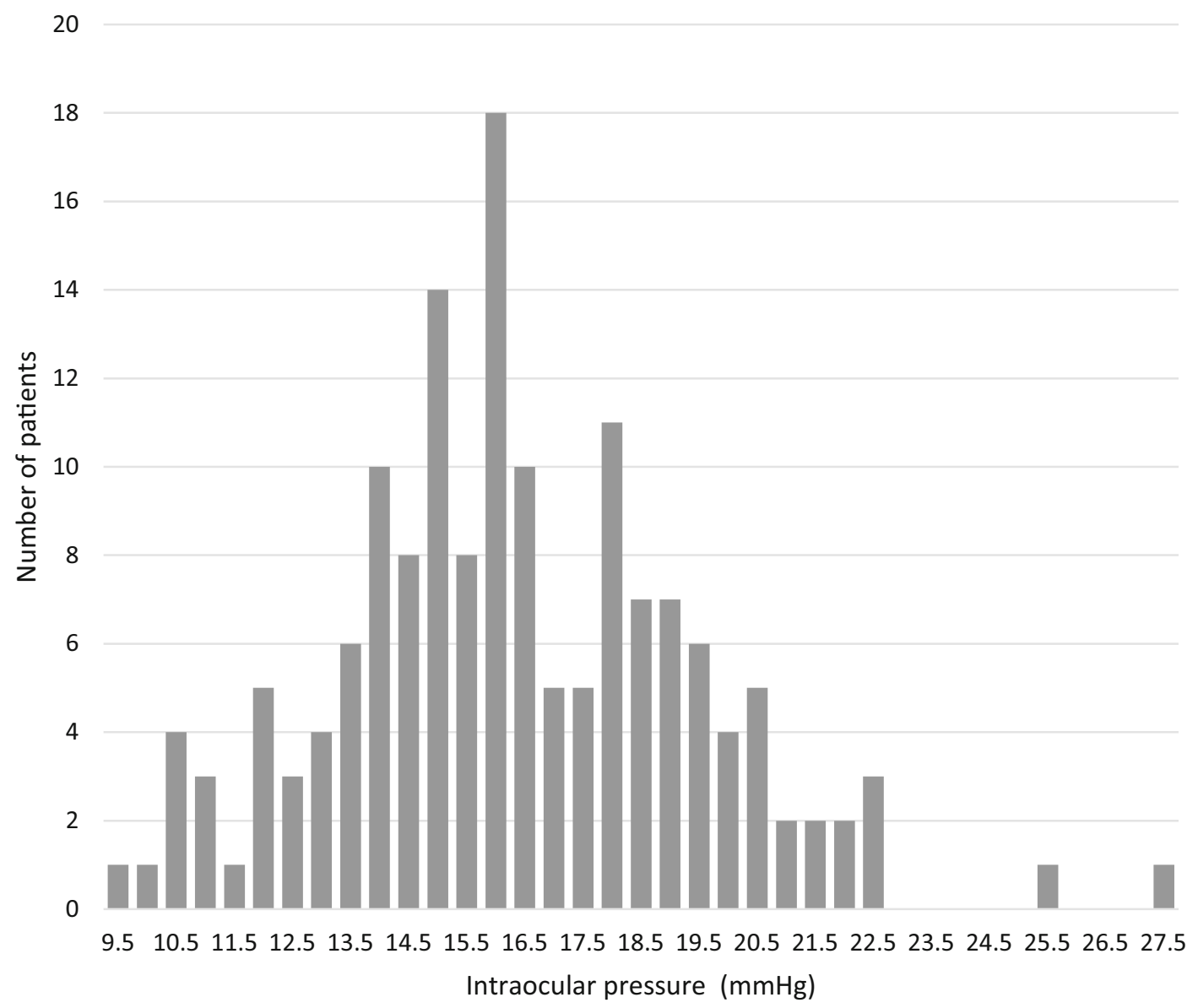

Fig. 1 Distribution of baseline intraocular pressure

patients were graded as moderate and included blepharal pigmentation, eye pruritus, eye irritation, and increased blood pressure. Blepharal pigmentation was reported during the 4-week visit together with skin itching and Duotrav was discontinued at the patient's request. Eye pruritus and eye irritation occurred at 4 and 14 days of treatment, respectively, and increased blood pressure was noted during the 4-week visit together with mild rash. In these three cases, treatment was discontinued at the doctor's discretion and all the events improved or resolved during the study period. Among the mild events observed, increased blurred vision (occurring at 8 weeks in one patient) and deepening of the eyelid sulcus and blepharoptosis (occurring at 17 days in one patient) led to treatment discontinuation at the doctor's discretion (Table 4).

All 157 eyes with at least 4 weeks of follow-up underwent serial examinations for eyelash changes, blepharal pigmentation, and deepening of the eyelid sulcus, where a change from baseline was considered an adverse event. The cumulative incidence was highest for eyelash changes (42 patients; $26.8 \%$ ), followed by blepharal pigmentation $(29 ; 18.5 \%)$ and deepening of the eyelid sulcus (13; 8.3\%; Table 5).

At 12 weeks, there were no significant changes from baseline in the mean systolic blood pressure $(135.3 \pm 17.4 \mathrm{mmHg}$ at baseline and $\quad 132.4 \pm 17.5 \mathrm{mmHg}$ at 12 weeks; $P=0.4859)$ diastolic blood pressure 
25

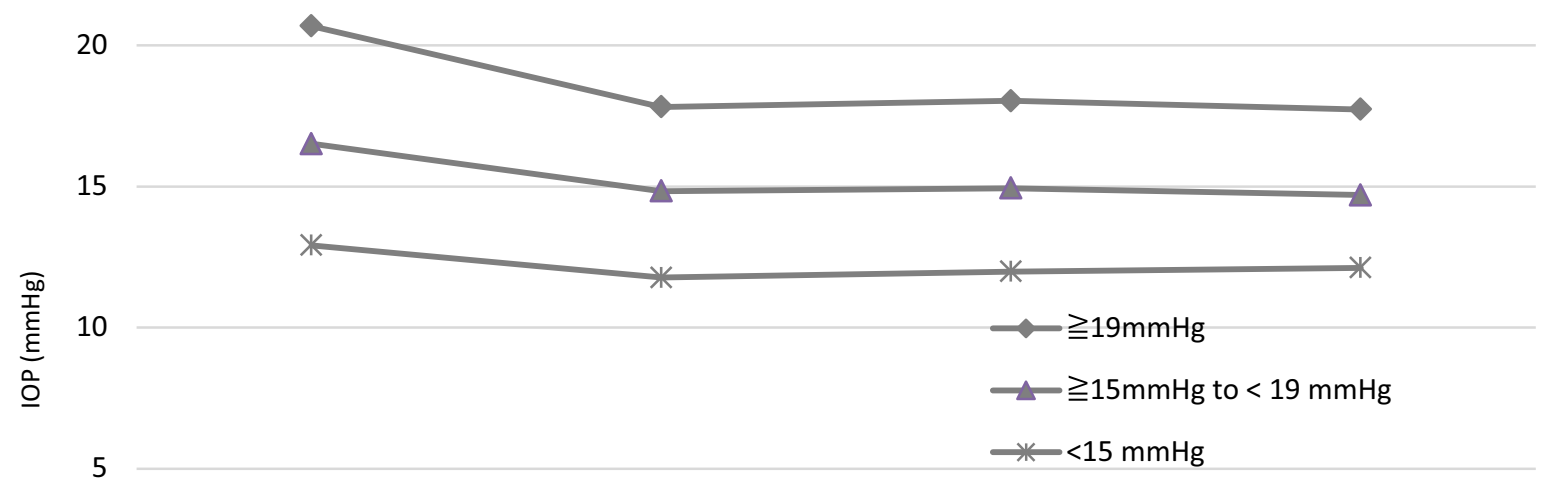

0

4 weeks $\quad 8$ weeks 12 weeks

\begin{tabular}{cccccc}
\hline Baseline IOP & & Baseline & 4 weeks & 8 weeks & 12 weeks \\
\hline$\geq 19 \mathrm{mmHg}, n=33$ & Mean $(\mathrm{mmHg})$ & 20.7 & 17.8 & 18.0 & 17.7 \\
& $\mathrm{SD}(\mathrm{mmHg})$ & 1.9 & 2.9 & 3.6 & 3.7 \\
& $P$ value* vs. baseline & & 0.0001 & $<0.0001$ & $<0.0001$ \\
\hline$\geq 15 \mathrm{mmHg}$ to & Mean $(\mathrm{mmHg})$ & 16.5 & 14.8 & 14.9 & 14.7 \\
$<19 \mathrm{mmHg}, n=78$ & $\mathrm{SD}(\mathrm{mmHg})$ & 1.2 & 2.3 & 2.3 & 2.1 \\
& $P$ value* vs. baseline & & $<0.0001$ & $<0.0001$ & $<0.0001$ \\
\hline$<15 \mathrm{mmHg}, n=46$ & Mean $(\mathrm{mmHg})$ & 12.9 & 11.8 & 12.0 & 12.1 \\
& SD (mmHg) & 1.5 & 1.8 & 2.2 & 1.8 \\
& $P$ value* vs. baseline & & 0.0324 & 0.0083 & 0.0005 \\
\hline
\end{tabular}

Fig. 2 Intraocular pressure reductions after switching by baseline intraocular pressure subgroups. Asterisks repeated-measures analysis of variance and Steel's multiple comparison test. IOP intraocular pressure, $S D$ standard deviation

$(80.2 \pm 11.1 \quad$ and $\quad 78.4 \pm 10.8 \mathrm{mmHg}$; $P=0.5266)$ or pulse rate $(70.8 \pm 13.0$ and $68.2 \pm 12.1 \mathrm{bpm} ; \quad P=0.3033) \quad$ among 151 patients with available data.

\section{DISCUSSION}

In patients with POAG, NTG, or OH whose IOP had been lowered with PGA monotherapy but who were judged to require an additional ophthalmic medication, switching to travoprost/timolol maleate fixed combination (Duotrav) produced a further reduction in IOP after 12 weeks of treatment and was not associated with serious adverse events.

The goal of treating glaucoma is to maintain visual function. Currently, IOP-lowering therapy is the only evidence-based treatment and to prevent the progression of visual field defects, a greater IOP-lowering effect is needed [21, 22]. 
Table 2 Multiple regression analysis

\begin{tabular}{llccr}
\hline Factor & Degree of freedom & Sum of squares & $\boldsymbol{F}$ value & $\boldsymbol{P}$ value \\
\hline PGAs & 3 & 106.7065789 & 8.26 & $<0.0001$ \\
Age & 1 & 1.1326213 & 0.26 & 0.6089 \\
Sex & 1 & 5.9941339 & 1.39 & 0.2402 \\
Dosing period of PGAs & 1 & 0.0018124 & 0.00 & 0.9837 \\
Baseline intraocular pressure & 1 & 68.9170780 & 16.00 & 0.0001 \\
Baseline MD value & 1 & 6.8635484 & 1.59 & 0.2090 \\
Baseline PSD value & 1 & 11.7128012 & 2.72 & 0.1014 \\
\hline
\end{tabular}

MD mean deviation, $P G A$ s prostaglandin analogs, PSD pattern standard deviation

In a multiple regression analysis with IOP reduction from baseline at 12 weeks after switching to Duotrav as a dependent variable, and demographic and baseline characteristics as independent variables, the type of PGA used in prior treatment and baseline IOP had a significant association. Coefficient of determination $\left[R^{2}\right]$ was 0.29

Table 3 Changes in scores of SPK and conjunctival hyperemia over time

\begin{tabular}{lllll}
\hline Scale & Baseline & 4 weeks & 8 weeks & 12 weeks \\
\hline SPK total scores & $0.58 \pm 1.31$ & $0.90 \pm 1.68$ & $0.88 \pm 1.78$ & $0.92 \pm 1.76$ \\
$P$ value* $^{*}$ vs. baseline & - & 0.1259 & 0.1616 & 0.1819 \\
Conjunctival hyperemia scores & $0.41 \pm 0.64$ & $0.52 \pm 0.69$ & $0.45 \pm 0.61$ & $0.49 \pm 0.63$ \\
$P$ value $^{*}$ vs. baseline & - & 0.3470 & 0.8215 & 0.3774 \\
\hline
\end{tabular}

Data are shown as mean \pm standard deviation

SPK Superficial punctate keratopathy

* Steel's test

Among current topical ophthalmic medications used for the treatment of glaucoma, PGAs are the most effective in lowering IOP and are regarded as the first-line treatment of choice. If the IOP reduction achieved with first-line monotherapy is insufficient, combination therapy using multiple drugs is indicated [3,4] and in such cases, fixed drug may be more advantageous for continuous treatment as they cause less events that might lead to early discontinuation [5-7]. Although previous studies reported reductions in IOP after switching from PGA monotherapy to fixed drug containing the corresponding PGA, few studies have evaluated the efficacy of switching from any PGA monotherapy to a specific fixed drug. Among fixed drugs currently available, only Duotrav contains polyquaternium-1 (Polyquad) as a preservative instead of BAK and therefore patients are not exposed to the compound. These considerations led us to design a study to evaluate the efficacy and safety of switching to Duotrav in patients maintained on monotherapy with one of the four PGAs currently available in Japan (travoprost, latanoprost, tafluprost, and bimatoprost).

In the present study, patients had a mean IOP of $16.3 \pm 3.1 \mathrm{mmHg}$ at baseline, which was calculated from measurements at two visits 
Table 4 Adverse events

\begin{tabular}{|c|c|c|c|c|c|c|}
\hline & & Adverse & & & & \\
\hline & & Mild & & Moderate & & Total \\
\hline Patients included in safety analysis & & 166 & & 166 & & 166 \\
\hline Number of events (number of cases) & & $38(22)$ & & $4(4)$ & & $42(26)$ \\
\hline Type & $n$ & $\%$ & $n$ & $\%$ & $n$ & $\%$ \\
\hline Eye disorders & 34 & - & 3 & - & 37 & - \\
\hline Eye lid sulcus deepened & 8 & 4.8 & & & 8 & 4.8 \\
\hline Growth of eyelashes & 5 & 3 & & & 5 & 3 \\
\hline Blepharal pigmentation & 3 & 1.8 & 1 & 0.6 & 4 & 2.4 \\
\hline Superficial punctate keratopathy & 4 & 2.4 & & & 4 & 2.4 \\
\hline Dry eyes & 3 & 1.8 & & & 3 & 1.8 \\
\hline Foreign body sensation in eyes & 2 & 1.2 & & & 2 & 1.2 \\
\hline Eyelid ptosis & 1 & 0.6 & & & 1 & 0.6 \\
\hline Eye irritation & 1 & 0.6 & 1 & 0.6 & 2 & 1.2 \\
\hline Eye pruritus & 1 & 0.6 & 1 & 0.6 & 2 & 1.2 \\
\hline Iritis & 1 & 0.6 & & & 1 & 0.6 \\
\hline Keratitis & 1 & 0.6 & & & 1 & 0.6 \\
\hline Vision blurred & 1 & 0.6 & & & 1 & 0.6 \\
\hline Visual acuity reduced & 1 & 0.6 & & & 1 & 0.6 \\
\hline Conjunctival hyperemia & 1 & 0.6 & & & 1 & 0.6 \\
\hline Corneal epitheliopathy & 1 & 0.6 & & & 1 & 0.6 \\
\hline Other & 4 & - & 1 & - & 5 & - \\
\hline Blood pressure increased & & & 1 & 0.6 & 1 & 0.6 \\
\hline Headache & 1 & 0.6 & & & 1 & 0.6 \\
\hline Herpes zoster & 1 & 0.6 & & & 1 & 0.6 \\
\hline Itchy skin & 1 & 0.6 & & & 1 & 0.6 \\
\hline Rash & 1 & 0.6 & & & 1 & 0.6 \\
\hline
\end{tabular}

during PGA monotherapy. It is not surprising that the IOP before adding a second drug was within the normal range [17] because NTG is dominant in Japan [23]. After switching to Duotrav, IOP significantly decreased from baseline at all specified timepoints during the 12-week follow-up period (4, 8, and 12 weeks) with the mean IOP decreasing to $14.6 \pm 3.2 \mathrm{mmHg}$ at 12 weeks. This corresponded to a mean $10.1 \%$ reduction from the baseline value. PGA therapy reduced IOP by more than $20 \%$ from pretreatment values even in patients with NTG $[24,25]$. The $10 \%$ reduction observed in this study after 
Table 5 Cumulative incidence rates of eyelash changes, blepharal pigmentation, and deepening of the eyelid sulcus

\begin{tabular}{llll}
\hline Adverse event & 4 weeks & 8 weeks & 12 weeks \\
\hline Eyelash change, $n(\%)$ & $21(13.4 \%)$ & $30(19.1 \%)$ & $42(26.8 \%)$ \\
Blepharal pigmentation, $n(\%)$ & $19(12.1 \%)$ & $26(16.6 \%)$ & $29(18.5 \%)$ \\
Deeping of the eyelid sulcus, $n(\%)$ & $7(4.5 \%)$ & $9(5.7 \%)$ & $13(8.3 \%)$ \\
\hline
\end{tabular}

switching to Duotrav is similar to those reported previously in NTG populations [26, 27]. The absolute reduction of about $1.5 \mathrm{mmHg}$ associated with switching to Duotrav seems to represent a meaningful treatment benefit, considering the findings from the Early Manifest Glaucoma Trial where every $1 \mathrm{mmHg}$ of IOP reduction has patient benefit [21].

Because individual baseline IOP values showed a wide variation, we assessed the treatment effect using baseline IOP values. When study eyes were divided into three groups according to the baseline IOP ( $\geq 19 \mathrm{mmHg}, \quad \geq 15$ to $<19 \mathrm{mmHg}$, or $<15 \mathrm{mmHg}$ ), all groups had significant IOP reductions from baseline at 4,8 , and 12 weeks after switching to Duotrav. Previous studies evaluating the efficacy of switching to fixed drug therapy were conducted primarily in eyes with a baseline IOP of $\geq 20 \mathrm{mmHg}$ or in those with $\geq 19 \mathrm{mmHg}$ for studies of Duotrav [10, 12, 13]. In the present study, significant IOP reductions were observed in eyes with a baseline IOP of $<19 \mathrm{mmHg}$ and even in those with $<15 \mathrm{mmHg}$, suggesting that NTG patients with normal IOP who experience a progression of visual field defects despite PGA monotherapy might also be good candidates for Duotrav therapy.

During the study, close monitoring was performed for SPK and conjunctival hyperemia, the most common side effects of PGA-containing ophthalmic treatments. Side effects were scored according to the NEI classification system and with reference to sample pictures. After the initiation of Duotrav therapy, SPK and conjunctival hyperemia scores showed no significant worsening over time. Based on these results, the effects of Duotrav on the ocular surface were considered minimal in the present study population, including dry eyes. SPK has been reported at a higher frequency in patients using timolol ophthalmic solution [28, 29] and might be caused by the presence of BAK in the timolol solution [30, 31]. This preservative has strong antimicrobial activity but adversely affects the ocular surface [32, 33]. Adverse effects on the ocular surface may therefore be reduced by choosing a BAK-free regimen [20, 34]. Duotrav is a fixed-combination ophthalmic solution of travoprost and timolol and free of BAK, whereas combination regimens using separate ophthalmic solutions of travoprost and timolol or using other fixed drugs are associated with exposure to BAK because except for travoprost, they all contain BAK. Duotrav contains polyquaternium-1 an alternative that is less toxic to the cornea than BAK [35]. There have been few studies on the effects of polyquaternium-1 in clinical practice but the present study suggests the advantage of using this compound as a preservative.

No serious adverse events were reported during Duotrav therapy but adverse events in six patients led to treatment discontinuation either at the patient's request or at the doctor's discretion, clearly suggesting that individual 
patients should be closely monitored. The cumulative incidence of eyelash changes, blepharal pigmentation and deepening of the eyelid sulcus increased throughout the study period. This suggests that it is important to instruct patients to remove any solution spilled on the eyelid skin by wiping or washing their face after the administration of Duotrav. There were no significant changes from baseline in the mean blood pressure or pulse rate after treatment did not change significantly.

This study had some limitations. The number of cases analyzed was relatively small, especially when the cases were divided into three groups, and using a larger number of patients would increase the statistical power. However, the results observed in this study showed a significant reduction of IOP. The open label and no control design might also introduce unconscious bias into some of the findings. The lack of washout phase before switching to Duotrav may be considered another limitation of this study. Therefore, we analyzed the IOP reduction from baseline at 12 weeks using multiple regression analysis and previous treatment was likely to be completely washed out at 12 weeks.

\section{CONCLUSIONS}

Duotrav appears to be a useful treatment as it exhibited a good IOP-lowering effect regardless of pretreatment IOP, and was associated with few adverse events. However, a multiple regression analysis identified baseline IOP and the type of PGA used in prior treatment as significant predictors of the magnitude of IOP reduction. This indicates that the magnitude of the effect of Duotrav is influenced by the pretreatment IOP; however, further analysis is required to determine the effect of PGA type. In the second report of this study, we will describe the analysis of our data by prior PGA monotherapy.

\section{ACKNOWLEDGMENTS}

This study was conducted with funding and support from the Japan Association of Health Service and Alcon Japan. Ltd. The article processing charges and open access fee for this publication were funded by Alcon Japan Ltd. All named authors meet the International Committee of Medical Journal Editors (ICMJE) criteria for authorship for this manuscript, take responsibility for the integrity of the work as a whole, and have given final approval to the version to be published. All authors had full access to all of the data in this study and take complete responsibility for the integrity of the data and accuracy of the data analysis. The authors would like to thank J. Ludovic Croxford, who provided English language editing services on behalf of inScience Communications. This assistance was funded by Alcon Japan Ltd.

Disclosures. Tadashi Nakano received consultant fees from Topcon Corporation and lecture fees from Alcon Japan Ltd, Santen Pharmaceutical Co., Ltd, Otsuka Pharmaceutical Co., Ltd, R-Tech Ueno Ltd, Senju Pharmaceutical Co., Ltd, Carl Zeiss Co., Ltd, Kaken Pharmaceutical Co., Ltd, MSD K.K., and Pfizer Japan Inc. Shiro Mizoue has received lecture fees from Alcon Japan Ltd, Pfizer Japan Inc., MSD K.K, NIDEK Co., Ltd, Senju Pharmaceutical Co., Ltd, Santen Pharmaceutical Co., Ltd, KOWA Pharmaceutical Co., Ltd, R-Tech Ueno Ltd, and Kaken Pharmaceutical Co., Ltd. Nobuo Fuse has received lecture fees from Alcon 
Japan Ltd, Santen Pharmaceutical Co., Ltd, Pfizer Japan Inc., R-Tech Ueno Ltd, and MSD K.K. Aiko Iwase has received consultant fees from Topcon Corporation and fees for expert testimony from KOWA Pharmaceutical Co., Ltd, payment for clinical trial from Heidelberg Engineering, Inc. and lecture fees from Alcon Japan Ltd, Santen Pharmaceutical Co., Ltd, Carl Zeiss Co., Ltd, Senju Pharmaceutical Co., Ltd, Otsuka Pharmaceutical Co., Ltd, WAKAMOTO Co., Ltd, and Pfizer Japan Inc. Shun Matsumoto has received lecture fees from Alcon Japan Ltd, Santen Pharmaceutical Co., Ltd, and Kaken Pharmaceutical Co., Ltd. Keiji Yoshikawa has received fees for expert testimony from Santen Pharmaceutical Co., Ltd, and lecture fees from Alcon Japan Ltd, Santen Pharmaceutical Co., Ltd, Pfizer Japan Inc., Otsuka Pharmaceutical Co., Ltd, R-Tech Ueno Ltd, Kaken Pharmaceutical Co., Ltd, Senju Pharmaceutical Co., Ltd, KOWA Pharmaceutical Co., Ltd, and MSD KK. The authors report no other conflicts of interest related to this work.

Compliance with ethics guidelines. All procedures followed were in accordance with the ethical standards of the responsible committee on human experimentation (institutional and national) and with the Helsinki Declaration of 1964, as revised in 2013. Informed consent was obtained from all patients for being included in the study.

Open Access. This article is distributed under the terms of the Creative Commons Attribution-NonCommercial 4.0 International License (http://creativecommons.org/licenses/ by-nc/4.0/), which permits any noncommercial use, distribution, and reproduction in any medium, provided you give appropriate credit to the original author(s) and the source, provide a link to the Creative Commons license, and indicate if changes were made.

\section{REFERENCES}

1. Rahman MQ Abeysinghe SS, Kelly S, et al. Persistence of glaucoma medical therapy in the Glasgow Glaucoma Database. Br J Ophthalmol. 2011;95:966-70.

2. Werner EB, Drance SM. Progression of glaucomatous field defects despite successful filtration. Can J Ophthalmol. 1977;12:275-80.

3. Kass MA, Heuer DK, Higginbotham EJ, et al. The Ocular Hypertension Treatment Study: a randomized trial determines that topical ocular hypotensive medication delays or prevents the onset of primary-open angle glaucoma. Arch Ophthalmol. 2002;120:701-13.

4. McCarty CA, Mukesh BN, Kitchner TE, et al. Intraocular pressure response to medication in a clinical setting: the Marshfield Clinic Personalized Medicine Research Project. J Glaucoma. 2008;17:372-7.

5. Djafari F, Lesk MR, Harasymowycz PJ, Desjardins D, Lachaine J. Determinants of adherence to glaucoma medical therapy in a long-term patient population. J Glaucoma. 2009;18:238-43.

6. Sleath B, Robin AL, Covert D, Byrd JE, Tudor G, Svarstad B. Patient-reported behavior and problems in using glaucoma medications. Ophthalmology. 2006;113:431-6.

7. Chrai SS, Makoid MC, Eriksen SP, Robinson JR. Drop size and initial dosing frequency problems of topically applied ophthalmic drugs. J Pharm Sci. 1974;63:333-8.

8. Higginbotham EJ. Considerations in glaucoma therapy: fixed combinations versus their component medications. Clin Ophthalmol. 2010;4:1-9.

9. Dunker S, Schmucker A, Maier H, Latanoprost/ Timolol Fixed Combination Study Group. Tolerability, quality of life, and persistency of use in patients with glaucoma who are switched to the fixed combination of latanoprost and timolol. Adv Ther. 2007;24:376-86.

10. Costa VP, Moreira H, Paolera MD, de Moraes Silva MRB. Efficacy and safety of travoprost $0.004 \% /$ timolol $0.5 \%$ fixed combination as transition therapy in patients previously on 
prostaglandin analog monotherapy. Clin Ophthalmol. 2012;6:699-706.

11. Paranhos A, Mendonça M, Silva MJ, et al. Hyperemia reduction after administration of a fixed combination of bimatoprost and timolol maleate to patients on prostaglandin or prostamide monotherapy. J Ocul Pharmacol Ther. 2010;26:611-5.

12. Pfeiffer N, Scherzer ML, Maier H, et al. Safety and efficacy of changing to the travoprost/timolol maleate fixed combination (Duotrav ${ }^{\circledR}$ ) from prior mono- or adjunctive therapy. Clin Ophthalmol. 2010;4:459-66.

13. Arend $\mathrm{KO}$, Raber TJ. Observational study results in glaucoma patients undergoing a regimen replacement to fixed combination travoprost $0.004 \% /$ timolol $0.5 \%$ in Germany. Ocul Pharmacol Ther. 2008;24:414-20.

14. Collaborative Normal-Tension Glaucoma Study Group. The effectiveness of intraocular pressure reduction in the treatment of normal-tension glaucoma. Am J Ophthalmol. 1998;126:498-505.

15. Kondo N, Sawada A, Yamamoto T, Taniguchi T. Correlation between individual differences in intraocular pressure reduction and outflow facility due to latanoprost in normal-tension glaucoma patients. Jpn J Ophthalmol. 2006;50:20-4.

16. Ang GS, Kersey JP, Shepstone L, Broadway DC. The effect of travoprost on daytime intraocular pressure in normal tension glaucoma: a randomised controlled trial. Br J Ophthalmol. 2008;92:1129-33.

17. The Japan glaucoma society guidelines for glaucoma. 3rd ed. Nippon Ganka Gakkai Zasshi. 2012;116:3-46 (in Japanese).

18. Holló G, Vuorinen J, Tuominen J, Huttunen T, Ropo A, Pfeiffer N. Fixed-dose combination of tafluprost and timolol in the treatment of open-angle glaucoma and ocular hypertension: comparison with other fixed-combination products. Adv Ther. 2014;31:932-44.

19. Kaur IP, Lal S, Rana C, Kakkar S, Singh H. Ocular preservatives: associated risks and newer options. Cutan Ocul Toxicol. 2009;28:93-103.

20. Yamazaki S, Nanno M, Kimura T, Suzumura $H$, Yoshikawa K. Effects of switching to SofZia-preserved travoprost in patients who presented with superficial punctate keratopathy while under treatment with latanoprost. Jpn J Ophthalmol. 2010;54:7-14.

21. Leske MC, Heijl A, Hussein M, Bengtsson B, Hyman L, Komaroff E, Early Manifest Glaucoma Trial
Group. Factors for glaucoma progression and the effect of treatment: the early manifest glaucoma trial. Arch Ophthalmol. 2003;121:48-56.

22. The AGIS Investigators. The Advanced Glaucoma Intervention Study (AGIS): 7. The relationship between control of intraocular pressure and visual field deterioration. Am J Ophthalmol. 2000;130:429-40.

23. Iwase A, Suzuki Y, Araie M, et al. The prevalence of primary open-angle glaucoma in Japanese: the Tajimi Study. Ophthalmology. 2004;111:1641-8.

24. Mizoue S, Nakano T, Fuse N, Iwase A, Matsumoto S, Yoshikawa K. Travoprost with sofZia ${ }^{\circledR}$ preservative system lowered intraocular pressure of Japanese normal tension glaucoma with minimal side effects. Clin Ophthalmol. 2014;8:347-54.

25. Cheng JW, Cai JP, Wei RL. Meta-analysis of medical intervention for normal tension glaucoma. Ophthalmology. 2009;116:1243-9.

26. Kanamoto T, Kiuchi Y, Ichioka I, et al. Clinical efficacy of travoprost-timolol fixed combination on ocular hypotensive agents and ocular surface agents in Japanese glaucoma patients. Nihon Ganka Gakkai Zasshi. 2013;117:793-8 (in Japanese).

27. Igarashi R, Togano T, Sakaue Y, Yoshino T, Ueda J, Fukuchi T. Effect on intraocular pressure of switching from latanoprost and travoprost monotherapy to timolol fixed combinations in patients with normal-tension glaucoma. J Ophthalmol. 2014;2014:720385.

28. Van Buskirk EM. Adverse reactions from timolol administration. Ophthalmology. 1980;87:447-50.

29. Rosin LM, Bell NP. Preservative toxicity in glaucoma medication: clinical evaluation of benzalkonium chloride-free $0.5 \%$ timolol eye drops. Clin Ophthalmol. 2013;7:2131-5.

30. Ishibashi T, Yokoi N, Kinoshita S. Comparison of the short-term effects on the human corneal surface of topical timolol maleate with and without benzalkonium chloride. J Glaucoma. 2003;12:486-90.

31. Baudouin C. Side effects of antiglaucomatous drugs on the ocular surface. Curr Opin Ophthalmol. 1996;7:80-6.

32. Burstein NL. Corneal cytotoxicity of topically applied drugs, vehicles and preservatives. Surv Ophthalmol. 1980;25:15-30.

33. Cha SH, Lee JS, Oum BS, Kim CD. Corneal epithelial cellular dysfunction from benzalkonium chloride 
(BAC) in vitro. Clin Exp Ophthalmol. 2004;32:180-4.

34. Aihara M, Oshima $H$, Araie M, EXTraKT study group. Effects of SofZia-preserved travoprost and benzalkonium chloride-preserved latanoprost on the ocular surface-a multicentre randomized single-masked study. Acta Ophthalmol. 2013;91:e7-14.
35. Ammar DA, Noecker RJ, Kahook MY. Effects of benzalkonium chloride- and polyquad-preserved combination glaucoma medications on cultured human ocular surface cells. Adv Ther. 2011;28:501-10. 